\title{
A Novel Method of Brain Tumor Segmentation using Extensive Feature Set
}

\author{
M.Y.Bhanumurthy ${ }^{1}$ and Koteswararao Anne ${ }^{2}$ \\ ${ }^{1}$ Dept.of ECE, Vasireddy Venkatadri Institute of Technology, Guntur-522006, A.P, INDIA; \\ ${ }^{2}$ Dean Academics, V.R.Siddhartha Engineering College, Vijayawada, A.P, INDIA; \\ mybhanu@gmail.com; raoanne@gmail.com
}

\begin{abstract}
Brain tissue Segmentation from the MRI images is having significance in the medical research field. The accurate Segmentation of the normal as well as the abnormal tissues is the complex assignment in this process. Because of the inconsistency and difficulty of abnormal tissues, MRI Brain Image Segmentation turned into more hard procedure. In this paper, a technique is proposed for segmenting the abnormalities such as Tumor and Atrophy in the MRI Brain images. (1) Feature extraction (2) Classification (3) Segmentation are the three stages offered in this work. At first, the features such as energy, entropy, homogeneity, contrast and correlation from MRI Brain Images are extracted. Next, by utilizing Neuro-Fuzzy classifier, the Classification process is carried out and for this process, the feature set is specified as the input. From the outcome of Classification, the images are categorized into normal as well as abnormal. The further procedure Segmentation is performed according to this outcome only. The abnormal MRI images are segmented into abnormal tissues like Tumor and Atrophy using Region Growing method. Utilizing MATLAB platform the implementation of the proposed technique is made. The experimentation is carried out on the MRI Brain Images by BrainWeb data sets. The performance of our proposed technique is assessed with the help of the metrics namely FPR, FNR, Specificity, Sensitivity and Accuracy. Therefore, using our proposed technique with enhanced classification, the abnormal tissues of MRI Brain images are segmented accurately.
\end{abstract}

Keywords:- Segmentation, Classification, Neuro-Fuzzy Logic, Normal and abnormal tissues, Region Growing method.

\section{Introduction}

The brain is the frontal most part of the central nervous system. It forms the Central Nervous System (CNS) along with the spinal cord. The Cranium, a bony box in the skull guards it. Because of our brain in practical we do lots of things like, to think, act, reason, walk, talk, the list is never-ending. Brain Tumors are one of the syndrome caused in the brain. In an (MR) images processing. As, in a number of neurological disorders like multiple sclerosis (MS) and Alzheimer's disease, the volume changes in total brain, WM, and GM can give major notification about neuronal and axonal loss [16].

The remaining of the paper is prepared as follows: After Introduction, the next section surveys several works which describes various techniques for segmenting the tissues in the MRI Brain images. Section 3 explains our proposed technique of segmenting the abnormal tissues of Brain MRI (BMRI) images. The outcomes regarding the performance of our proposed work are specified in the Section 4 and as a final point; our paper is summed up with the conclusion part in Section 5. 


\section{Literature Survey}

Researchers proposed for many researches for the brain image segmentation. A short access of several researches is offered here. Arnaldo Mayer and Hayit Greenspan [17] have offered an automated segmentation framework for brain MRI volumes based on adaptive mean-shift grouping in the joint spatial and intensity feature space. The technique was authorized both on simulated and real brain datasets, and the outcomes were compared with state-of-the-art algorithms. The benefits over intensity based GMM EM schemes as well as additional state-of-the-art techniques were established. Moreover they proved that by means of the AMS framework, segmentation of the normal tissues is not degraded by the presence of abnormal tissues. The algorithm gave good outcomes on noisy and biased data while only a rudimental bias field improvement part executed and no spatial prior was extracted from an atlas. And thanks to the adaptive mean-shift ability to work with non-convex clusters in the joint spatial intensity feature space and also the mean-shift noise smoothing behavior.

Mert R. Sabuncu et al. [18] have examined a generative model that guides to label fusion style image segmentation techniques. They originated several algorithms that merge transmitted training labels into a single segmentation estimate in the proposed framework. An expert gave a dataset of 39 brain MRI scans and equivalent label maps and we analytically compared these segmentation algorithms with Free Surfer's broadly-used atlas-based segmentation tool. Their outcomes established that the proposed framework yields an accurate and robust segmentation tools that are employed on large multi-subject datasets. They utilized one of the enhanced segmentation algorithms to calculate hippocampal volumes in MRI scans of 282 subjects, in a second experiment. A assessment of these measurements across clinical and age groups signifies that the proposed algorithms were adequately sensitive to detect hippocampal volume variations related with earlier Alzheimer's disease and aging.

By utilizing a subject-specific tissue probabilistic atlas produced from longitudinal data, Feng Shi et al. [19] have offered a framework for presenting neonatal brain tissue segmentation. Proposed method has received the benefit of longitudinal imaging study in their system, i.e., by means of the segmentation outcomes of the images obtained at a late time to direct the segmentation of the images obtained at neonatal stage. Compared to the two population-based atlases the testing outcomes revealed that the subject-specific atlas has better performance. And moreover the proposed algorithm attained comparable performance as manual raters in neonate brain image segmentation. By attaining optimal segmentation results in a broad range of $0.3-0.6$, the atlas sharpness parameter has been shown robust appearance. For the selection of late time-point image, the segmentation accuracy remains alike when the atlas was developed by either one-year-old or two-year-old image.

Juin-Der Lee et al. [20] have offered the most statistical segmentation methods in the literature and have presumed that either the intensity allocation of every tissue variety was Gaussian, or the logarithmic transformation of the raw intensity was Gaussian. As an alternative of setting up further classes to model "mixels," they proposed a power transformation approach to carry out automatic segmentation of brain MR images into CSF, GM, and WM. By instinct it was understandable that the familiar Box-Cox power transformation model was capable to give a statistically significant and helpful solution to proposed difficulty. To include both Gaussian intensity distributions as well as non-Gaussian distributions, the shape parameter utilized to widen the traditional Gaussian mixture models. And the parameters can be expected by means of the EM algorithm. They authorized the approach against four real and simulated datasets of normal brains from the IBSR and BrainWeb. 
Testing's on real data from the IBSR have shown that compared with other techniques utilized presently, the proposed approach attains higher Jaccard indexes. The power transformation approach maintains the simplicity of the Gaussian mixtures, and in addition it has the prospective to simplify the multivariate versions personalized for segmentation by means of multi-modality images.

Dalila Cherifi et al. [21] have illustrated normal tissue's recognition than tumor extraction (applied for GBM and MS diseases). To detach the abnormal tissues they have offered brain recognition techniques. Based on thresholding utilized for tumor extraction (GBM and MS diseases) they have proposed and applied the technique. They have originated that the local thresholding provides a good outcomes comparing with the others. They have accomplished that when they merge median filter, local thresholding and post processing in such a way that the resultant algorithm is tougher. For tissue recognition and tumor extraction they have executed categorization based on EM segmentation technique. Comparing with thresholding particularly for detecting the small regions of necrotizing tissue which was inside Anaplastic cells (pseudo-Palisading necrosis) for GBM tissue, proposed technique provided us better outcomes; and it mainly for the reason that of parameters that utilized in this algorithm.

Nagesh Vadaparthi et al. [22] have offered a paper in which particular cases like Acoustic neuroma, it was presumed that there was an option of hearing loss, dizziness and other symptoms associated to brain. Surgery can cure various acoustic neuromas. Hence, it was required to segment the image more correctly, which assisted to recognize the damaged tissues to be repaired and can be corrected by surgery. And so a new novel segmentation algorithm based on Skew Gaussian distribution was proposed in proposed paper, which assisted to recognize the tissues more correctly. Because of the basic structure of Skew Gaussian distribution it was suitable for symmetric and asymmetric distribution. The performance evaluation was succeeded by utilizing quality metrics. The outcomes proved that, proposed developed algorithm outperforms the existing algorithm. Various models were exploited to recognize the diseases, although due to the utility of non-ionizing radiation, MRI brain segmentation has achieved popularity over the other models.

Usually noise is generated by equipments, environment and also the performance of operator in MRI Brain images which creates serious incorrectness in the outcome of Segmentation procedure. Several of the unverified techniques did not deal with the intensity and in-homogeneity artifacts. And also the managed techniques undergo with the shortcomings of manual intervention for providing a priori notification. Pathological tissues demonstrate inconsistency in their structures. The shape of these tissues is deformable, the location of them across the patients may differ extensively, and also their characteristics of texture and intensity might vary. These difficulties of the existing schemes are generally un-solvable. A few techniques do not consider the large deformation of brain structures. The practice of brain atlas might show the way to false learning, though such deformations occurs.

\section{Proposed Methodology}

Initially, the BMRI images are given as input to our proposed work and the feature sets are extracted from these input images. From these feature sets, the images are classified into two kinds of tissues - normal and abnormal using the Neuro-Fuzzy classifier. Then the abnormal tissues Tumor and Atrophy are segmented using Region Growing Method. The proposed work is illustrated in Fig. 1.

\subsection{Phases of Neuro-Fuzzy based segmentation}

For our proposed method, to segment the BMRI images effectively, the three phases are presented which are as follows: 
M.Y.Bhanumurthy \& Koteswararao Anne; A Novel Method of Brain Tumor Segmentation using extensive feature set, Advances in Image and Video Processing, Volume 2 No 5, Oct (2014); pp: 11-26

I. Feature set Extraction

II. Neuro-Fuzzy classifier based Classification

III. Classified tissue's Segmentation

\subsubsection{Phase I: Extraction of extensive feature sets}

In order to classify the given Brain MRI images, the features from these MRI images are initially extracted. In our work, the statistical features such as Energy, Entropy, Homogeneity, Contrast and Correlation are extracted from these input BMRI images.

\section{Energy}

Energy is also called as uniformity. Within $[0,1]$ the range of energy is presented. The value of energy for a constant image is 1 . The equation for finding energy is,

$$
E_{g}=\sum_{i, j} p(i, j)^{2}
$$

where, $p(i, j)$ is the pixel value at the point $i, j$ of the BMRI image of size $M \times N$.

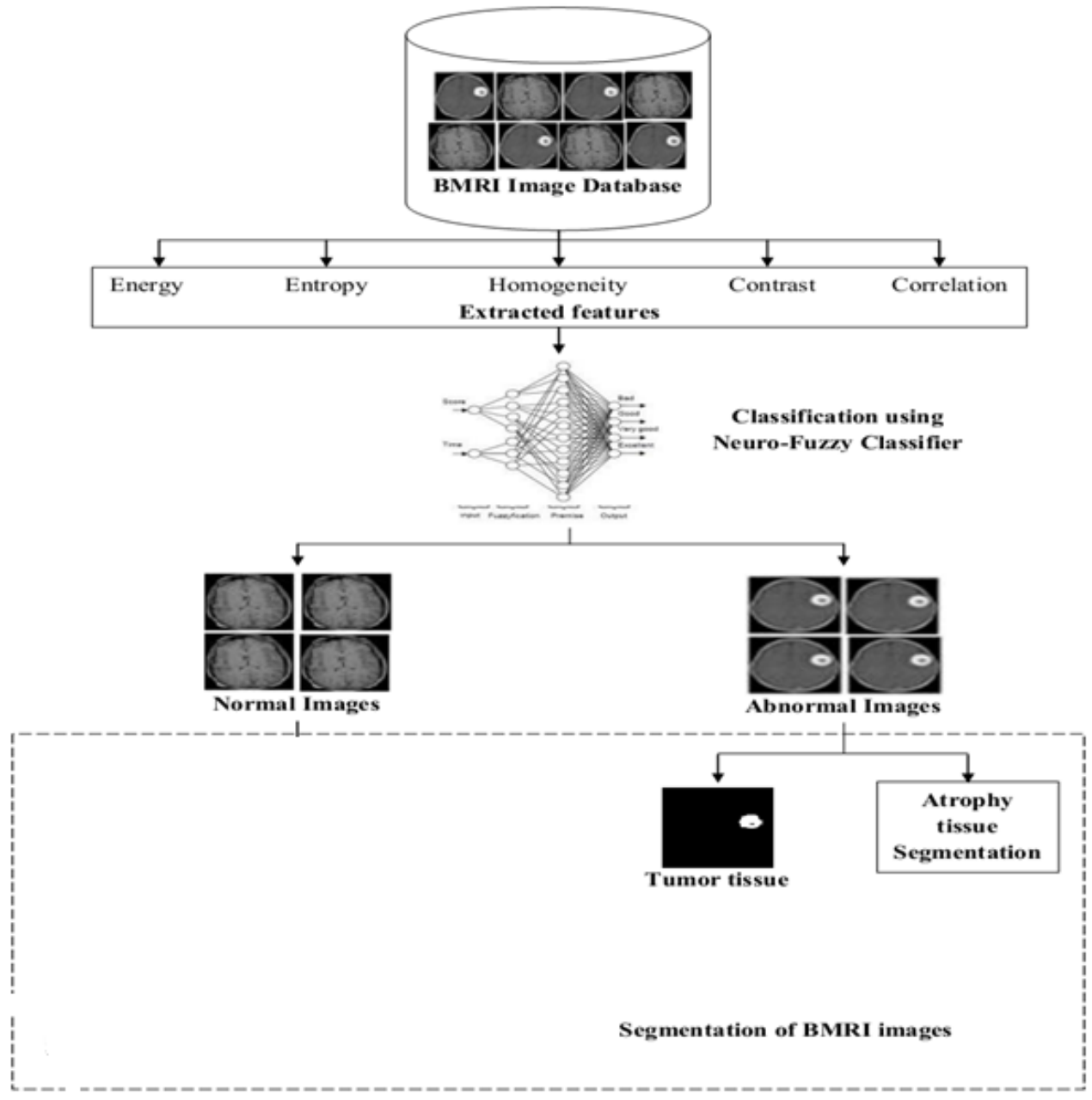

\section{Entropy}

Figure 1: Proposed Neuro-Fuzzy based segmentation block diagram

Entropy helps to characterize the texture of the BMRI image and to find out the distribution variation in a region of the image. Entropy is calculated as follows, 


$$
E_{p}=\sum_{k=0}^{G-1} P b_{k}\left(\log _{2} P b_{k}\right)
$$

where, $P b_{k}$ is the probability of $k^{\text {th }}$ gray level and the $k^{\text {th }}$ gray level is calculated using $\frac{Z_{k}}{M \times N}$. In this, $Z_{k}$ represents the total number of pixels in the image with $\mathrm{k}^{\text {th }}$ gray levels. $G$ indicates the total number of gray levels.

\section{Homogeneity}

Homogeneity provides the closeness of the elements. It has the range of $[0,1]$. It is computed as,

$$
H_{m}=\sum_{i, j} \frac{p(i, j)}{1+|i-j|}
$$

\section{Contrast}

The intensity contrast between a pixel of an image and the neighbor of that pixel throughout the whole image is defined by this Contrast measure. For a constant image, the contrast is set as 0 . It is specified as,

$$
C_{n}=\sum_{i, j}|i-j|^{2} p(i, j)
$$

\section{Correlation}

It tells about the correlation between a pixel and its neighbor over the whole BMRI image. Its range is $[-1,1]$. If an image has the value of correlation as 1 means, then it indicates the perfectly positively correlated image and if it is -1 means, then it shows the image is perfectly negatively correlated. The correlation of a constant image is not a number.

$$
C_{r}=\sum_{i, j} \frac{\left(i-\mu_{i}\right)\left(j-\mu_{j}\right) p(i, j)}{\sigma_{i} \sigma_{j}}
$$

where, $\mu_{i}, \mu_{j}, \sigma_{i}, \sigma_{j}$ are the means and standard deviations of the partial probability density functions $P_{i}, P_{j}$.

Mean,

$$
\mu=\frac{1}{M N} \sum_{i=1}^{M} \sum_{j=1}^{N} p(i, j)^{2}
$$

Standard Deviation, $\quad \sigma=\sqrt{\frac{1}{M N} \sum_{i=1}^{M} \sum_{j=1}^{N}(p(i, j)-\mu)^{2}}$

Variance,

$$
\operatorname{Var}=\sqrt{\sigma}
$$

Thus the feature correlation of the images is calculated using the mean and variance equations. Hence, all the feature sets $E_{g}, E_{p}, H_{m}, C_{n}$, and $C_{r}$ are extracted from the input BMRI images directly. 


\subsubsection{Phase II: Classification using Neuro-fuzzy classifier}

The BMRI images are classified using the Neuro-Fuzzy classifier. The extracted features $E_{g}, E_{p}, H_{m}, C_{n}$, and $C_{r}$ are given as the input to the Neuro-Fuzzy Classifier for classifying all the given BMRI images into 2 classes such as Normal BMRI images and Abnormal BMRI images. The Neuro-fuzzy system has a three-layered architectural design; the following diagram fig. 2 shows the basic structure of the neuro-fuzzy classifier system. Neuro-Fuzzy classifier is a fuzzy based system that is trained by a learning algorithm derived from Neural Networks. The learning algorithm only performs on the local information and provides the local modifications in the fuzzy system. In general, a neuro-fuzzy system generates very powerful solutions instead of using the system components individually.

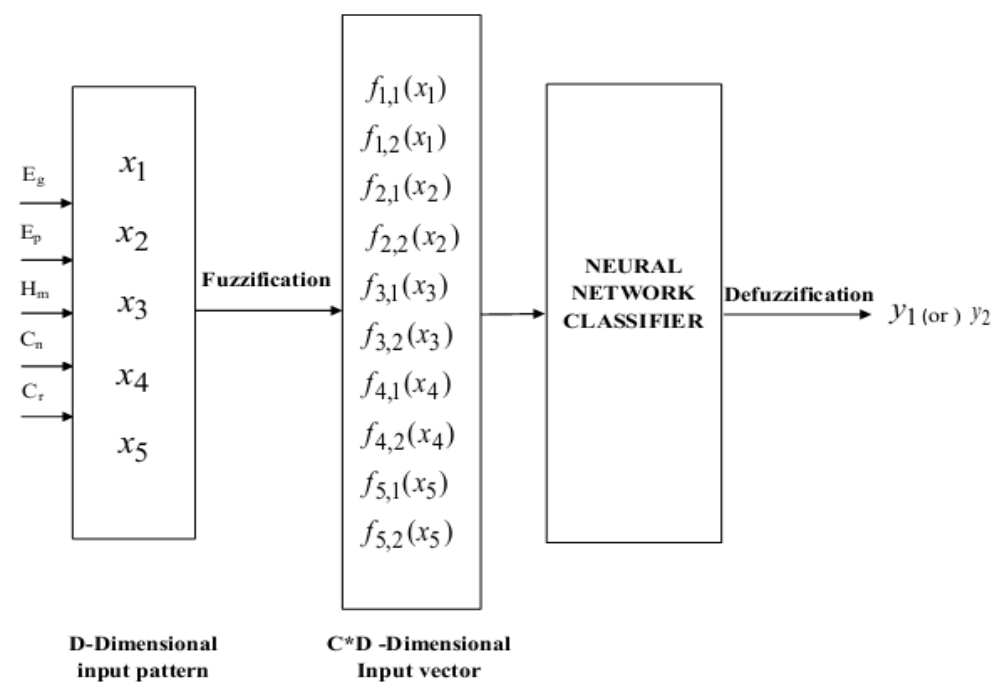

Figure 2: Architecture of Neuro-Fuzzy classifier

\section{Fuzzification}

The input values are the extracted features $E_{g}, E_{p}, H_{m}, C_{n}$, and $C_{r}$, which are received by the system as the input and then these input feature values are fuzzified using membership functions (MF) that facilitates the membership of each features to different classes. The hidden and interrelated information are extracted from the features to the classes through the MF, which leads to get more accuracy of the classification phase using Neuro-fuzzy classifier. The membership matrix comprises with 5 rows and 2 columns, in which the number of rows is equal to the number of features and the number of columns is equal to the number of classes.

The membership matrix $f_{d, c}\left(x_{d}\right)$ produced, describes the degree of belonging of different features $(D)$ to different classes $(C)$.

Where, $x_{d}-d^{\text {th }}$ feature value of pattern $X$.

$d-1,2, \ldots, D$, here number of features is 5 .

$c-1,2, \ldots, C$, here number of classes is 2 .

The representation of pattern is as follows,

$$
X=\left[x_{1}, x_{2}, x_{3}, x_{4}, x_{5}\right]^{T}
$$


In this a $\pi$-type MF is used as the membership function to classify the images. It is a bounded function having a shape similar to that of the Gaussian/exponential function. The $\pi$-type MF has fuzzifier $(m)$ as the parameter that can be tuned corresponding to the need of the problem. This controls the generalization capability by choosing a proper value of the fuzzifier $m$ and gives more flexibility for classifying the images. The steepness of the Gaussian function is controlled by varying the fuzzifier value, which is defined as follows,

$$
\pi(X ; a, r, b)= \begin{cases}0, & \text { if } X \leq a \\ 2^{m-1}\left[\frac{(X-a)}{(r-a)}\right]^{m}, & \text { if } a<X \leq p \\ 1-2^{m-1}\left[\frac{(r-X)}{(r-a)}\right]^{m}, & \text { if } p<X \leq r \\ 2^{m-1}\left[\frac{(X-r)}{(b-r)}\right]^{m}, & \text { if } r<X \leq q \\ 1-2^{m-1}\left[\frac{(b-X)}{(b-r)}\right]^{m}, & \text { if } q<X<b \\ 0, & \text { if } X \geq b\end{cases}
$$

The value $r$ is the center of MF, and $r=\frac{(p+q)}{2}$, in which $p$ and $q$ are the two crossover points. The membership function after the fuzzification process is expressed for a pattern $X$ as follows,

$$
F(X)=\left[\begin{array}{ll}
f_{1,1}\left(x_{1}\right) & f_{1,2}\left(x_{1}\right) \\
f_{2,1}\left(x_{2}\right) & f_{2,2}\left(x_{2}\right) \\
f_{3,1}\left(x_{3}\right) & f_{3,2}\left(x_{3}\right) \\
f_{4,1}\left(x_{4}\right) & f_{4,2}\left(x_{4}\right) \\
f_{5,1}\left(x_{5}\right) & f_{5,2}\left(x_{5}\right)
\end{array}\right]
$$

All rows and columns in the membership matrix are cascaded and converted into a vector by this cascading. This generated vector $V_{i}$ is given as the input to the Neural Network (NN).

\section{Neural Network}

In this, Feed Forward Multi-layer Perceptron classifier is used which has three layers such as input layer, hidden layer and output layer.

The total number of input nodes of the NN is equal to the product of the number of features and classes. In this paper the product of 5 features and 2 classes is 10 , which is the number of input nodes of the NN. The total number of output nodes from the NN is same as that of the number of classes, and here 2 output nodes are generated from the NN. The total number of hidden nodes is equal to the square root of the product, of the number of input nodes and output nodes. The structure for the Neural Network is given in figure 3. 


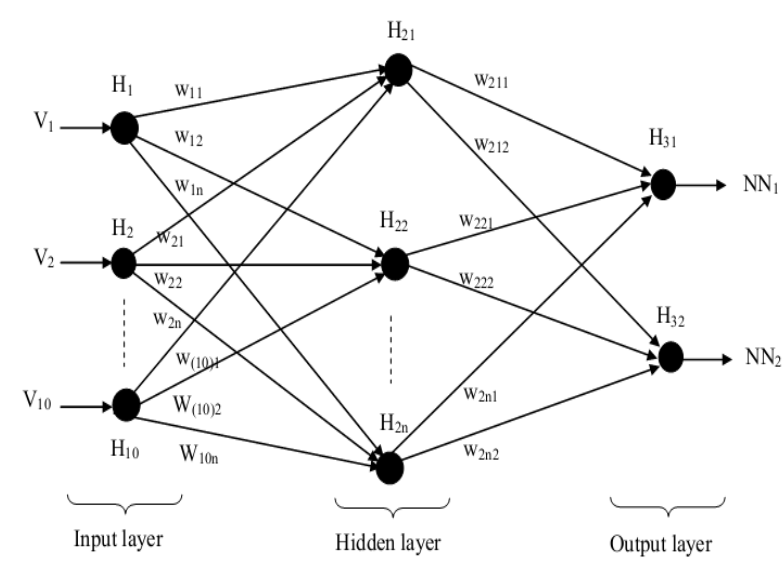

Figure 3: Neural Network classifier

\section{Defuzzification}

Then the defuzzification process is carried out on the output nodes of NN, by performing a MAX (maximum) operation. The output is a single value, $y_{1}$ or $y_{2}$ for a given BMRI image. From this value, we can able to classify whether the given input BMRI image is normal or abnormal.

\subsubsection{Segmentation of classified tissues}

Utilizing Neuro-fuzzy classifier the BMRI images are classified and after that the images are comprised only in any of the two different images normal and abnormal or pathological images. From the abnormal images, tumor and atrophy are segmented.

\section{Segmentation of Tumor}

For segmenting the tumor tissues, the classified abnormal images are utilized. Region Growing Method (RGM) is used for the segmentation of tumor tissues. RGM is one of the image segmentation techniques, in which the first seed points are chosen from the abnormal images. For the neighboring pixels in the first seed points, the neighbor pixels are checked out to verify the neighboring pixels are located within the region or not [23]. Accordingly, the tumor tissue part is segmented from the abnormal image and the resultant image is $I_{T}$.

\section{Segmentation of Atrophy}

By using the White Matter and Gray Matter ratio as the whole brain, the degree of atrophy is established from the abnormal image. This is the way to identify the atrophy in the beginning stage.

\section{Atrophy Ratio (AR)}

The Atrophy Ratio (AR) is computed by comparing the White Matter and Gray Matter with the whole size of the brain, which includes White Matter, Gray Matter and Cerebro-Spinal Fluid. For a BMRI image, the atrophy ratio is calculated as follows:

$$
A R=\frac{(W M+G M)}{(W M+G M+C S F)}
$$

In this above equation (9), WM, GM and CSF specify the region area of White Matter, Gray Matter and Cerebro-Spinal Fluid respectively. We can tell the atrophy level in the brain, according to the ratio of Atrophy. It is studied that the existence of atrophy is in high level, if the ratio value is small. From the Atrophy ratio, the diseases like multiple sclerosis, Alzheimer's disease, Pick's disease, senile dementia, vascular dementia, stroke, etc. [24] are identified. 


\section{Atrophy Factor (AF)}

Atrophy Factor is a measurement that helps out to study the Atrophy Rate among two consecutive check-ups from the BMRI images for the same patient within a specific time interval. The AF is computed as specified in the below equation (10).

$$
A F=\frac{A_{t 1}-A_{t 2}}{A_{t 1}}
$$

In the above equation (10), $A_{t 1}$ and $A_{t 2}$ are the two consecutive MRI checkups within a specific time interval for the Atrophy of whole brain. In general, the difference in time is one year. We can say that no Atrophy is obtainable throughout this year, if the resultant AF value is too small or almost zero. If the value of AF is improved, then the level of Atrophy in brain is as well high. Therefore, the tumor and atrophy segmentation are efficiently made from the categorized abnormal BMRI images.

\section{$4 \quad$ Results and Discussions}

Our proposed Neuro-Fuzzy based segmentation for the effective segmentation of tumor and atrophy is implemented using the MATLAB platform on the Brain MRI images from the dataset. The data set description is given below in detail.

\subsection{Dataset Description:}

BrainWeb dataset is utilized with different BMRI images for our proposed work. Based on standard tissue segmentation mask, BrainWeb datasets give MRI brain images with unreliable image quality. The datasets are too based on an anatomical structure of a normal brain, which results from the tasks of registering and preprocessing of 27 scans from the same individual with segmentation. Different kinds of tissues are well identified in this dataset, both the types of tissue memberships "fuzzy" and "crisp" are assigned to each voxel. The sample Brain MRI images from the BrainWeb data set are specified in the figure 4 given below.

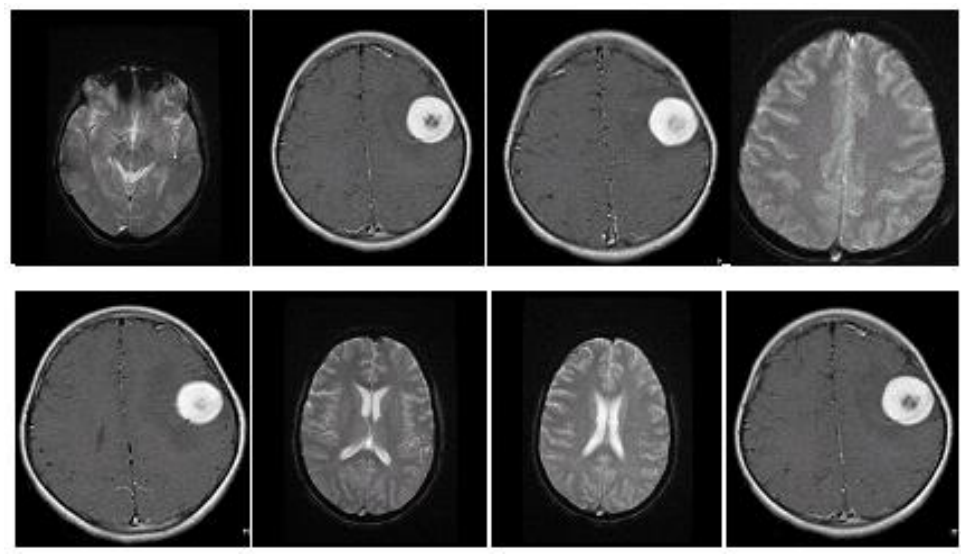

Figure 4: Sample BMRI images from dataset

Our proposed work is estimated by means of 23 BrainWeb MRI images. 9 images are normal and the remaining 14 images are abnormal among 23 MRI images. At first, the BMRI images from this dataset is taken and offered to the procedure of our proposed NFBIS. Five of the statistical characteristics from these BMRI images are extracted. Then for the categorization of images these extracted features are utilized. In order to categorize the particular images into normal and abnormal images, Neuro-Fuzzy classifier is utilized as the classifier in this proposed NFBIS work. The classified normal and abnormal images are specified in the

figure 5 given below. 

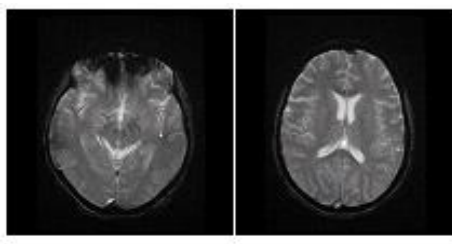

(a)
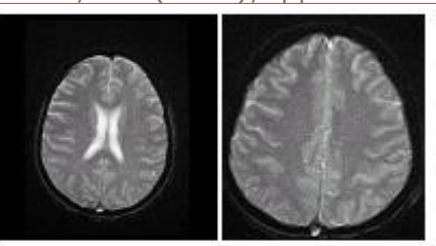

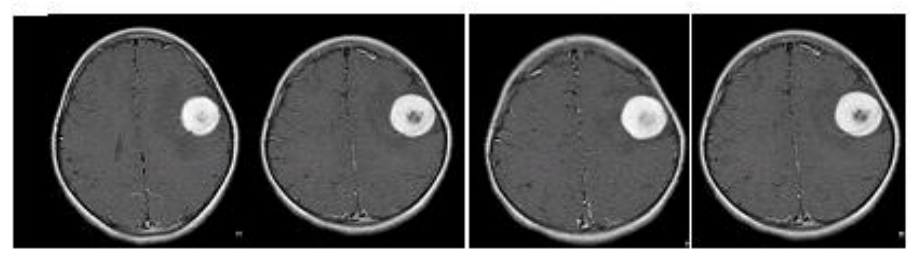

(b)

Figure 5: Normal and Abnormal Images

From the abnormal BMRI images, the abnormal tissues Tumor and Atrophy are segmented. By means of Region Growing Method, the segmentation of tumor is performed. The images of tumor tissues after segmentation are specified in the following figure 6.
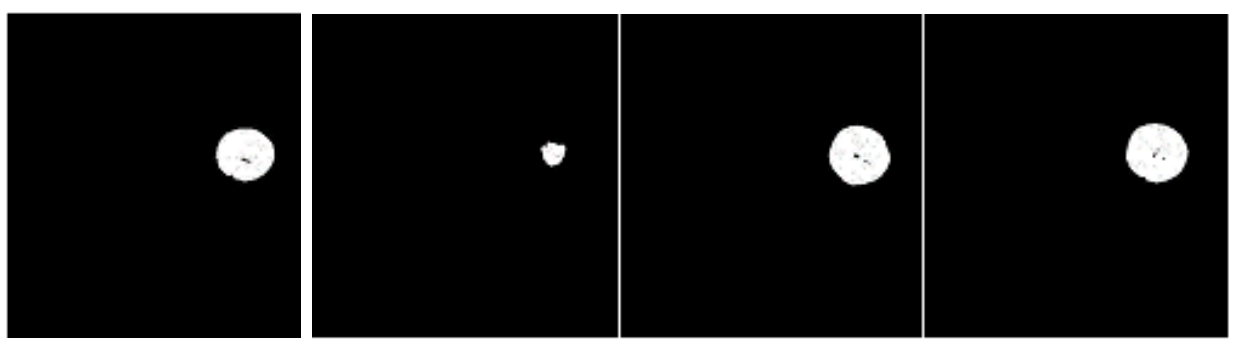

Figure 6: Segmentation of abnormal images

Table I provides the tabular values for the accuracy of abnormal tissue atrophy segmentation.

Table I: Atrophy level results for various images

\begin{tabular}{|l|c|}
\hline Images & Atrophy level (in \%) \\
\hline Image-1 & 0.790629155 \\
\hline Image-2 & 0.652814507 \\
\hline Image-3 & 0.875238649 \\
\hline Image-4 & 0.865906419 \\
\hline Image-5 & 0.966725302 \\
\hline
\end{tabular}

The image-5 has the extremely high atrophy level (96.67\%), which shows that the existence of atrophy is in low level. Next the existence of atrophy level is low level than the image 5 for the images 3 and 4 , as the values are $87.52 \%$ and $86.59 \%$, respectively. The existence of atrophy is high in image 2 and 1 , since both have the values $65.28 \%$ and $79.06 \%$ respectively.

\subsection{Performance Evaluation:}

By utilizing the performance measures namely False Positive Rate, False Negative Rate, Sensitivity, Specificity and Accuracy, the performance of the system is estimated. The basic count values such as True Positive (TP), True Negative (TN), False Positive (FP) and False Negative (FN) are used by these measures. Both the categorization of normal and abnormal images and the segmentation efficiency of abnormal images are examined by our proposed work, which are clarified in detail in the next sections. 


\subsubsection{Results of Classification Evaluation:}

The BrainWeb images include both the normal and abnormal images in our work. These images are categorized into normal and abnormal individually by the procedure of Neuro-Fuzzy classifier. The efficiency of the classifier is examined by the metrics False Positive Rate, False Negative Rate, Sensitivity, Specificity and Accuracy. The explanation of TP, TN, FP, FN values for the categorization of normal and abnormal images is specified in the table II given below.

Table II: Description of TP, TN, FP, FN values for the classification of normal and abnormal images

\begin{tabular}{|c|c|c|}
\hline Description & $\begin{array}{c}\text { Classified as } \\
\text { normal image }\end{array}$ & $\begin{array}{c}\text { Classified as } \\
\text { abnormal image }\end{array}$ \\
\hline Actually normal image & TP & FN \\
\hline Actually abnormal image & FP & TN \\
\hline
\end{tabular}

\section{False Positive Rate (FPR)}

The percentage of cases where an image was classified to normal images, but in fact it did not.

\section{False Negative Rate (FNR)}

$$
F P R=\frac{F P}{F P+T N}
$$

The percentage of cases where an image was classified to abnormal images, but in fact it did.

$$
F N R=\frac{F N}{F N+T P}
$$

\section{Sensitivity}

The proportion of actual positives which are correctly identified is the measure of the sensitivity. It relates to the ability of test to identify positive results.

$$
\text { Sensitivity }=\frac{\text { Number of true positives }}{\text { Number of true positives }+ \text { Number of false negatives }} \times 100
$$

\section{Specificity}

The proportion of negatives which are correctly identified is the measure of the specificity. It relates to the ability of test to identify negative results.

$$
\text { Specificit } y=\frac{\text { Number of true negatives }}{\text { Number of true negatives }+ \text { Number of false positives }} \times 100
$$

\section{Accuracy}

We can compute the measure of accuracy from the measures of sensitivity and specificity as specified below.

$$
\text { Accuracy }=\frac{T P+T N}{T P+T N+F P+F N} \times 100
$$

The subsequent table III explains the categorization efficiency outcomes for the normal and abnormal images with various metric values. 
M.Y.Bhanumurthy \& Koteswararao Anne; A Novel Method of Brain Tumor Segmentation using extensive feature set, Advances in Image and Video Processing, Volume 2 No 5, Oct (2014); pp: 11-26

Table III: Effectiveness of classification results using Neuro-Fuzzy classifier for the normal and abnormal images

\begin{tabular}{|c|c|}
\hline Metrics & Values \\
\hline TP & 8 \\
\hline TN & 14 \\
\hline FP & 0 \\
\hline FN & 1 \\
\hline FPR & 0 \\
\hline FNR & 6.667 \\
\hline Sensitivity & $88.9 \%$ \\
\hline Specificity & $100 \%$ \\
\hline Accuracy & $95.65 \%$ \\
\hline
\end{tabular}

In our proposed work, we can establish the efficiency of categorization for the normal and abnormal images by means of Neuro-Fuzzy classifier from the above table II. False Positive Rate and False Negative Rate values are 0 and 6.667, respectively, which explains that our proposed work has low error rate in categorizing images. Properly categorized percentages of normal images are specified by Sensitivity. Neuro-fuzzy classifier offers very high $(88.9 \%)$ values for the metric sensitivity, in which only one of the normal image is categorized as abnormal. In addition, Specificity is another metric that specifies the percentage of abnormal images properly categorized. The classifier provides $100 \%$ specificity rate by categorizing the entire actual abnormal images into abnormal images in our work. The highest value in sensitivity and specificity and also the lowest value in the error rates False Positive Rate and False Negative Rate open a mode to raise the categorization correctness outcome with the value $95.65 \%$. Therefore we can show that in proposed work categorization of BMRI BrainWeb images offers high classification accuracy.

\subsubsection{Results of Segmentation Evaluation:}

The abnormal tissues namely tumor and atrophy are segmented from the BMRI images of BrainWeb. The tumor segmentation outcomes are tabularized in the following table IV.

Table IV: Tumor segmentation results for various images

\begin{tabular}{|c|c|c|c|c|c|c|c|c|c|}
\hline Images & TP & TN & FP & FN & FPR & FNR & $\begin{array}{c}\text { Sensitivity } \\
\text { (in \%) }\end{array}$ & $\begin{array}{c}\text { Specificity } \\
\text { (in \%) }\end{array}$ & $\begin{array}{c}\text { Accuracy } \\
\text { (in \%) }\end{array}$ \\
\hline Img 1 & 5405 & 255045 & 1694 & 864 & 0.0065981 & 0.137821 & 86.217 & 99.34018 & 99.0274 \\
\hline Img 2 & 5405 & 255045 & 1694 & 864 & 0.0065981 & 0.137821 & 86.217 & 99.34018 & 99.0274 \\
\hline Img 3 & 3870 & 252820 & 5454 & 102601 & 0.0211171 & 0.963652 & 3.634 & 97.88828 & 70.3751 \\
\hline Img 4 & 5405 & 255045 & 1694 & 864 & 0.0065981 & 0.137821 & 86.217 & 99.34018 & 99.0274 \\
\hline Img 5 & 3870 & 252820 & 5454 & 102601 & 0.0211171 & 0.963652 & 3.634 & 97.88828 & 70.3751 \\
\hline
\end{tabular}

Corresponding graph of table IV is designed in Figure 7 with different BMRI images for the Tumor segmentation. The evaluation outcomes explains whether our proposed work is worked efficiently or not for the tumor segmentation. 


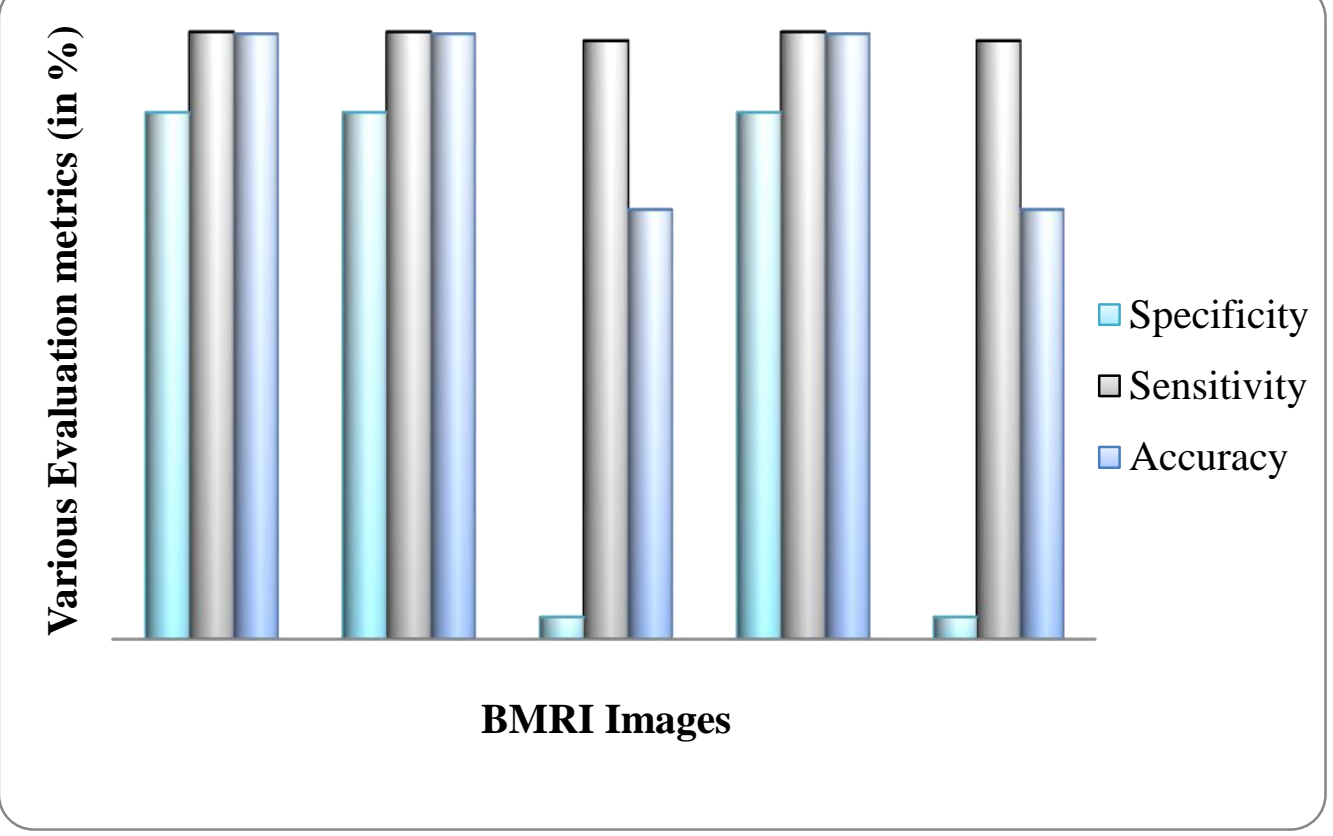

Figure 7: Sensitivity, Specificity and Accuracy results of Tumor segmentation

The outcomes of tumor segmentation are viewed with the metric values from the table IV and Figure 7. The abnormal tumor tissue segmentation of our proposed work presents improved accuracy outcomes of $99.027 \%, 99.027 \%, 70.375 \%, 99.027 \%$ and $70.375 \%$ for the images 1, 2, 3, 4 and 5, respectively. The outcomes of specificity give high values of $99.34 \%, 99.34 \%, 97.88 \%, 99.34 \%$ and $97.88 \%$ for the images $1,2,3,4$ and 5 . The raise in the specificity values builds the tumor segmentation very accurately. The sensitivity values for the images 1, 2 and 3 are high (86.217\%) and for the images 3 and 5 are very low (3.634\%). The low value in sensitivity is not a difficulty to obtain accuracy with improved outcome. In addition, our work provides only very low values for the error rates FPR and FNR. It's a great benefit for our work to get improved accuracy of segmentation. Correct tumor parts were only segmented from the abnormal BMRI images of our proposed work. This can be recognized from the high accuracy outcomes of tumor segmentation.

\subsubsection{Comparative Analysis for our proposed work with the existing works:}

For the categorization of normal and abnormal brain images our proposed work makes use of Neuro-Fuzzy classifier. We can establish that our proposed work helps to attain very good accuracy for the categorization of images utilizing Neuro-Fuzzy classifier from the above sections. And also we can establish this categorization accuracy outcome by comparing other classifiers. We have utilized Artificial Neural Network and Fuzzy C-Means for our comparison in our work. The comparison outcomes are presented in the following table V. 
M.Y.Bhanumurthy \& Koteswararao Anne; A Novel Method of Brain Tumor Segmentation using extensive feature set, Advances in Image and Video Processing, Volume 2 No 5, Oct (2014); pp: 11-26

Table V: Comparison results for the image classification with other classifiers

\begin{tabular}{|c|c|c|c|}
\hline Metrics & Fuzzy C-Means & $\begin{array}{c}\text { Artificial Neural } \\
\text { Network }\end{array}$ & $\begin{array}{c}\text { Neuro-Fuzzy in our } \\
\text { proposed work }\end{array}$ \\
\hline TP & 0 & 1 & 8 \\
\hline TN & 14 & 9 & 14 \\
\hline FP & 0 & 5 & 0 \\
\hline FN & 9 & 8 & 1 \\
\hline FPR & 0 & 0.3571 & 0 \\
\hline FNR & 0.3913 & 0.4706 & 6.667 \\
\hline Sensitivity (in \%) & 0 & 11.11 & 88.9 \\
\hline Specificity (in \%) & 100 & 64.29 & 100 \\
\hline Accuracy (in \%) & 60.87 & 43.48 & 95.65 \\
\hline
\end{tabular}

Below specified figure 8 explains the comparison outcomes of the classifiers for the BMRI image categorization with different metrics. The improved accuracy outcomes of categorization of BMRI images into normal and abnormal images are presented by our proposed work. In comparison with the classifier Neuro-Fuzzy, both the Fuzzy C-Means and Artificial Neural Networks gives very less accuracy values for the categorization of images. The sensitivity for the Fuzzy C-Means and Artificial Neural Networks are $0 \%$ and $11.11 \%$, which is low in compared with our classifier, Neuro-Fuzzy $88.9 \%$. The specificity is $100 \%$ for our classifier and for the Fuzzy C-means classifier. However the accuracy is $\mathbf{9 5 . 6 5 \%}$ for our Neuro-fuzzy classifier and the fuzzy C-means and ANN contain only low categorization accuracy results of $60.87 \%$ and $43.48 \%$, respectively. From these outcomes, it is known that by means of Neuro-Fuzzy classifier in our work provides very good for the categorization purpose as it gives improved accuracy outcomes. Therefore, our work shows that it is worth for the categorization and segmentation of BMRI images.

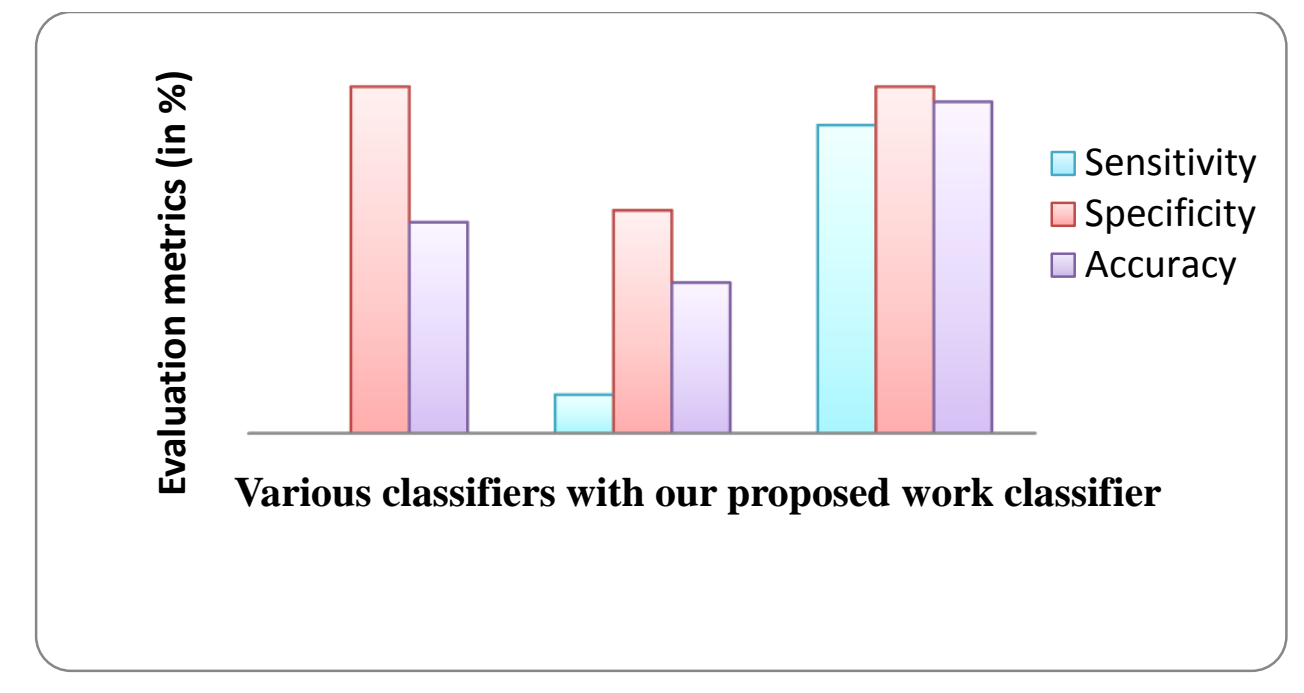

Figure 8: Comparison graph for the image classification with other classifiers

\section{Conclusion}

A Neuro Fuzzy based BMRI image segmentation technique with three phases - Feature Extraction, Classification and Segmentation was proposed in this paper. The features from the BMRI images were extracted and then specified to the Neuro-Fuzzy classifier. The classification of normal and abnormal images was made by this Neuro-Fuzzy classifier and these classified abnormal images were segmented efficiently by our proposed method. The testing was performed with the BrainWeb 
images dataset. The performance measures False Positive Rate, False Negative Rate, sensitivity, specificity and accuracy were evaluated for our proposed method. The testing was prepared for establishing the accuracy of both the classification of images into normal and abnormal and segmentation of abnormal tissue like Tumor. The efficiency of the classification of images is very high by presenting very good accuracy outcomes and also the segmentation of Tumor offers very accurate outcomes. From the outcomes, we have showed that the Neuro-Fuzzy classifier utilized in our proposed work outperforms the other classifiers Fuzzy C-Means and ANN by facilitated very good accuracy of $95.65 \%$ in categorizing the images into normal and abnormal.

\section{REFERENCES}

[1]. P. Hagmann, J.P. Thiran, L. Jonasson, "DTI mapping of human brain connectivity: statistical fibre tracking and virtual dissection", Neurolmage, 2003.

[2]. M.C. Davidson, K. M. Thomas. and B. J. Casey, "Imaging the developing brain with fMRI", Mental Retardation and developmental disabilities research reviews, 2003.

[3]. V. A. Grau, U. J. Mewes, M. Alcaniz, "Improved watershed transform for medical image segmentation using prior information", IEEE Trans. on Medical Imaging, 2004, 23(4): 447-458

[4]. H Lv., K. H. Yuan, S. L. Bao, "An eSnake model for medical imaging segmentation”, Progress in Natural Science, 2005.

[5]. D. L. Pham and J. L. Prince, "Adaptive fuzzy segmentation of magnetic resonance images," IEEE Trans. Med. Imag., 1999.

[6]. A. F. Goldszal, C. Davatzikos, D. L. Pham, M. X. H. Yan, et al, "An image processing system for qualitative and quantitative volumetric analysis of brain images," J. Comput. Assist. Tomogra., 1998.

[7]. Arnold J.B., Liow, J.-S., Schaper, K.A., et al., "Qualitative and quantitative evaluation of six algorithms for correcting intensity nonuniformity effects". Neurolmage, 2001.

[8]. R. Moller., R. Zeipelt. "Automatic segmentation of 3D-MRI data using a genetic algorithm, Medical Imaging and Augmented Reality", 2001. Proceedings. International Workshop on, 10-12 June 2001:278 - 281 .

[9]. W. M.Wells, III,W. E. L. Grimson, R. Kikinis. "Adaptive segmentation of MRI data”, IEEE Trans. Medical Imaging , 1996.

[10]. J. C. Bezdek, L.O. Hall, L. P. Clarke, "Review of MR image segmentation techniques using pattern recognition," Med. Phys., vol. 20, No. 4, pp. 1033-1048, 1993.

[11]. Suetens, E. Bellon, D. Vandermeulen, M. Smet, G. Marchal, J. Nuyts, L. Mortelman, "Image segmentation: methods and applications in diagnostic radiology and nuclear medicine," European Journal of Radiology, vol. 17, pp. 14-21, 1993.

[12]. A. Goshtasby, D. A. Turner, "Segmentation of Cardiac Cine MR Images for extraction of right and left ventricular chambers," IEEE sTrans. Med. Imag., vol. 14, No. 1, pp. 56-64, 1995. 
[13]. D. Brzakovic, X. M. Luo, P. Brzakovic, "An approach to automated detection of tumors in mammograms," IEEE Trans. Med. Imag., vol. 9, No. 3, pp. 233-241, 1990.

[14]. J. F. Brenner, J. M. Lester, W.D. Selles, "Scene segmentation in automated histopathology: techniques evolved from cytology automation," Pattern Recognition, vol. 13, pp. 65-77, 1981.

[15]. K. Lim, A. Pfefferbaum, "Segmentation of MR brain images into cerebrospinal fl-uid spaces, white and gray matter," J. Comput. Assist. Tomogr., vol. 13, pp. 588-593, 1989.

[16]. Zhang Y, Brady M, Smith S. "Segmentation of brain MR images through a hidden Markov random field model and expectation-maximization algorithm,". IEEE Trans Med. Imag., pp. 45-57, 2001.

[17]. Arnaldo Mayer and Hayit Greenspan, "An Adaptive Mean-Shift Framework for MRI Brain Segmentation", IEEE Transactions On Medical Imaging, Vol. 28, No. 8, August 2009.

[18]. Mert R. Sabuncu, B.T. Thomas Yeo, Koen Van Leemput, Bruce Fischl and Polina Golland, "A Generative Model for Image Segmentation Based on Label Fusion", leee Transactions On Medical Imaging, 2009.

[19]. Feng Shi, Yong Fan, Songyuan Tang, John H. Gilmore, Weili Lin, Dinggang Shen, "Neonatal brain image segmentation in longitudinal MRI studies", Elsevier Inc., 2009.

[20]. Juin-Der Lee, Hong-Ren Su, Philip E. Cheng*, Michelle Liou, John A. D. Aston, Arthur C. Tsai, and Cheng-Yu Chen, "MR Image Segmentation Using a Power Transformation Approach", IEEE Transactions On Medical Imaging, Vol. 28, No. 6, June 2009.

[21]. Dalila Cherifi, M.Zinelabidine Doghmane, Amine Nait-Ali , Zakia Aici,Salim Bouzelha, "Abnormal tissus extraction in MRI Brain medical images", IEEE, 2011.

[22]. Nagesh Vadaparthi, Srinivas Yarramalle, Suresh Varma Penumatsa, "Unsupervised Medical Image Segmentation On Brain MRI Images Using Skew Gaussian Distribution", IEEE-International Conference on Recent Trends in Information Technology, ICRTIT 2011.

[23]. Frank Y. Shih and Shouxian Cheng, "Automatic seeded region growing for color image segmentation", Journal of Image and Vision Computing, Vol. 23, pp. 877-886, 2005.

[24]. Rowayda A. Sadek, "An Improved MRI Segmentation for Atrophy Assessment", International Journal of Computer Science Issues, Vol. 9, No 2, pp. 569-574, May 2012. 